\title{
New Extensions of Some Known Special Polynomials under the Theory of Multiple q-Calculus
}

\author{
Mehmet Acikgoz ${ }^{1}$, Serkan Araci, ${ }^{2, *}$ Uğur Duran ${ }^{3}$
${ }^{1}$ Department of Mathematics, Faculty of Arts and Science, University of Gaziantep, Gaziantep, Turkey
${ }^{2}$ Department of Economics, Faculty of Economics, Administrative and Social Science, Hasan Kalyoncu University, Gaziantep, Turkey ${ }^{3}$ Department of Mathematics, Faculty of Arts and Science, University of Gaziantep, Gaziantep, Turkey *Corresponding author: mtsrkn@hotmail.com

\section{Received June 12, 2015; Revised September 25, 2015; Accepted October 03, 2015}

\begin{abstract}
In the year 2014, the idea of multiple q-calculus was formulated and introduced in the book of Nalci and Pashaev [9] in which this idea is simple but elegant method in order to derive new generating functions of some special polynomials that are generalizations of known $q$-polynomials. In this paper, we will use Nalci and Pashaev's method in order to find a systematic study of new types of the Bernoulli polynomials, Euler polynomials and Genocchi polynomials. Also we will obtain recursive formulas for these polynomials.
\end{abstract}

Keywords: Quantum calculus, Multiple quantum calculus, q-Bernoulli polynomials, q-Euler polynomials, q-Genocchi polynomials, Generating function.

Cite This Article: Mehmet Acikgoz, Serkan Araci, and Uğur Duran, "New Extensions of Some Known Special Polynomials under the Theory of Multiple q-Calculus.” Turkish Journal of Analysis and Number Theory, vol. 3, no.5 (2015): 128-139. doi: 10.12691/tjant-3-5-4.

\section{Introduction}

1.1. $q$-Calculus. The usual quantum calculus (or recalled $q$-calculus) has been extensively studied for a long time by many mathematicians, physicists and engineers. The development of $q$-calculus stems from the applications in many fields such as engineering, economics, math-ematics, and so on. One of the important branches of $q$-calculus is $q$-special polynomials. For example, Kim [18] constructed $q$-generalized Euler polynomials based on $q$-exponential function. Moreover, Srivastava et al investigated Apostol $q$-Bernoulli, Apostol $q$-Euler polynomials and Apostol $q$-Genocchi polynomials. This is why $q$-calculus is thought as one of the useful tools to study with special numbers and polynomias. For more information related these issues, see, e.g. [1,2,3,5,6,8-13,16-21].

Before starting at multiple $q$-calculus, we first give some basic notations about $q$-calculus which can be found in [3].

For a real number (or complex number) $x, q$-number (quantum number) is known as

$$
[x]_{q}:=\left\{\begin{array}{cc}
\frac{1-q^{x}}{1-q}, & \text { if } q \neq 1, \\
x, & \text { if } q=1
\end{array}\right.
$$

which is also called non-symmetrical $q$-number. The followings can be easily derived using (1.1):

$$
\begin{array}{r}
{[x+y]_{q}=[x]_{q}+q^{x}[y]_{q}(q \text {-addition formula) }} \\
{[x-y]_{q}=-q^{x-y}[y]_{q}+[x]_{q}(q \text {-substraction formula) }}
\end{array}
$$

$$
[x y]_{q}=[x]_{q}[y]_{q^{x}} \quad(q \text {-product rule })
$$

$$
\left[\frac{x}{y}\right]_{q}=\frac{[x]_{q}}{[y]_{q}^{\frac{x}{y}}}=\frac{[x]_{q}^{\frac{1}{y}}}{[y]_{q}^{\frac{1}{y}}} \text { (q-division rule) }
$$

where $x, y$ are real or complex numbers.

The $q$-binomial coefficients are defined for positive integer $n, k$ as

$$
\left[\begin{array}{l}
n \\
k
\end{array}\right]_{q}=\frac{[n]_{q} !}{[k]_{q} ![n-k]_{q} !}
$$

where $[n]_{q} !=[n]_{q}[n-1]_{q}[n-2]_{q} \ldots[1]_{q}, \quad n=1,2, \ldots$; $[0]_{q} !=1$.

The $q$-derivative $D_{q} f(x)$ of a function $f$ is given as

$D_{q} f(x)=\frac{f(x)-f(q x)}{(1-q) x}, q \neq 1, x \neq 0, D_{q} f(0)=f^{\prime}(0)$,

provided $f^{\prime}(0)$ exists.

For any $z \in \mathbb{C}$ with $|z|<1$,

$$
e_{q}(z)=\sum_{n=0}^{\infty} \frac{z^{n}}{[n]_{q} !} \text { and } E_{q}(z)=\sum_{n=0}^{\infty} q^{\left(\begin{array}{l}
n \\
2
\end{array}\right)} \frac{z^{n}}{[n]_{q} !} .
$$

For the $q$-commuting variables $x$ and $y$ such as $y x=q x y$, we know that

$$
e_{q}(x+y)=e_{q}(x) e_{q}(y) .
$$


The $q$-integral was defined by Jackson as follows:

$$
\int_{0}^{x} f(y) d_{q} y=(1-q) x \sum_{n=0}^{\infty} f\left(q^{n} x\right) q^{n}
$$

provided that the series on right hand side converges absolutely.

1.2. Multiple $q$-calculus. All notations and all corollaries written in this part have been taken from the Book of Nalci and Pashaev [9].

Consider basis vector $\vec{q}$ with coordinates $q_{1}, q_{2}, \ldots, q_{N}$ so that the multiple $q$-number can be de.ned as

$$
[n]_{q_{i}, q_{j}}:=\frac{q_{i}^{n}-q_{j}^{n}}{q_{i}-q_{j}}=[n]_{q_{j}, q_{i}},
$$

which is symmetric. Hence, we can write $N \times N$ matrix with $q$-numbers elements in the following form:

$$
\left([n]_{q_{i}, q_{j}}\right)=\left(\begin{array}{cccc}
{[n]_{q_{1}, q_{1}}} & {[n]_{q_{1}, q_{2}}} & \ldots & {[n]_{q_{1}, q_{N}}} \\
{[n]_{q_{2}, q_{1}}} & {[n]_{q_{2}, q_{2}}} & & {[n]_{q_{2}, q_{N}}} \\
\ldots & \ldots & \ldots & \ldots \\
{[n]_{q_{N}, q_{1}}} & {[n]_{q_{N}, q_{2}}} & \ldots & {[n]_{q_{N}, q_{N}}}
\end{array}\right)
$$

Diagonal terms of this matrix are defined in the limit $q_{j} \rightarrow q_{i}$ as

$$
\lim _{q_{j} \rightarrow q_{i}}[n]_{q_{i}, q_{j}}=\lim _{q_{j} \rightarrow q_{i}} \frac{q_{i}^{n}-q_{j}^{n}}{q_{i}-q_{j}}=n q_{i}^{n-1} .
$$

So, by (1.8), we see that this symmetric matrix can be shown as

$$
\left([n]_{q_{i}, q_{j}}\right)=\left(\begin{array}{cccc}
n q_{i}^{n-1} & {[n]_{q_{1}, q_{2}}} & \cdots & {[n]_{q_{1}, q_{N}}} \\
{[n]_{q_{2}, q_{1}}} & n q_{2}^{n-1} & & {[n]_{q_{2}, q_{n}}} \\
\ldots & \ldots & \ldots & \ldots \\
{[n]_{q_{N}, q_{1}}} & {[n]_{q_{N}, q_{2}}} & \cdots & n q_{N}^{n-1}
\end{array}\right) .
$$

The followings can be easily derived using (1.7):

$$
[n+m]_{q_{i}, q_{j}}=q_{i}^{n}[m]_{q_{i}, q_{j}}+q_{j}^{m}[n]_{q_{i}, q_{j}}
$$

( $q$-multiple addition formula)

$$
[n-m]_{q_{i}, q_{j}}=-q_{j}^{-m}\left([n]_{q_{i}, q_{j}}-q_{i}^{n-m}[m]_{q_{i}, q_{j}}\right)
$$

( $q$-multiple substraction formula)

$$
[n m]_{q_{i}, q_{j}}=[m]_{q_{i}, q_{j}}[n]_{q_{i}^{m}, q_{j}^{m}}
$$

(q-multiple product rule)

$$
\left[\frac{n}{m}\right]_{q_{i}, q_{j}}=\frac{[n]_{q_{i}, q_{j}}}{[m]_{q_{i}^{m}}^{\frac{n}{m}}, q_{j}^{\frac{n}{m}}}=\frac{[n]_{q_{i}^{\frac{1}{m}}, q_{j}^{\frac{1}{m}}}}{[m]_{q_{i}^{\frac{1}{m}}, q_{j}^{\frac{1}{m}}}^{\frac{1}{2}}}
$$

(q-multiple division rule)

where $n, m$ are real or complex numbers.
In multiple $q$-calculus, multiple $q$-derivative with base $q_{i}, q_{j}$ is given by

$$
\mathrm{D}_{q_{i}, q_{j}} f(x)=\frac{f\left(q_{i} x\right)-f\left(q_{j} x\right)}{\left(q_{i}-q_{j}\right) x}
$$

representing $N \times N$ matrix of multiple $q$-derivative operators $D:=\left(D_{q_{i}, q_{j}}\right)$ which is sym-metric: $D_{q_{i}, q_{j}}=D_{q_{j}, q_{i}}$ where $i$ and $j=1,2, \ldots, N$.

$$
D=\left(D_{q_{i}, q_{j}}\right)=\left(\begin{array}{cccc}
D_{q_{1}, q_{1}} & D_{q_{1}, q_{2}} & \ldots & D_{q_{1}, q_{N}} \\
D_{q_{2}, q_{1}} & D_{q_{2}, q_{2}} & \ldots & D_{q_{2}, q_{N}} \\
\ldots & \ldots & \ldots & \ldots \\
D_{q_{N}, q_{1}} & D_{q_{N}, q_{2}} & \ldots & D_{q_{N}, q_{N}}
\end{array}\right) .
$$

Corollary 1. For $N=1$ case and $q_{1}=q_{2} \equiv q$, we have

$$
[n]_{q, q}=n q^{n-1} \text { and } D_{q, q}=M_{q} \frac{d}{d x}
$$

where $M_{q}=q^{x \frac{d}{d x}}$. Also, in the case $q=1$, we have the standard number $[n]_{1,1}=n$ and the usual derivative $D_{1,1}=\frac{d}{d x}$.

Corollary 2. For $N=2$ case, we have

$$
\begin{aligned}
& {[n]_{q_{1}, q_{1}}=n q_{1}^{n-1},[n]_{q_{1}, q_{2}}=[n]_{q_{2}, q_{1}}=\frac{q_{1}^{n}-q_{2}^{x}}{q_{1}-q_{2}},} \\
& {[n]_{q_{2}, q_{2}}=n q_{2}^{n-1}} \\
& D_{q_{1}, q_{1}}=M_{q_{1}} \frac{d}{d x}, D_{q_{1}, q_{2}}=D_{q_{2}, q_{1}}=\frac{M_{q_{1}}-M_{q_{2}}}{\left(q_{1}-q_{2}\right) x}, \\
& D_{q_{2}, q_{2}}=M_{q_{2}} \frac{d}{d x}
\end{aligned}
$$

Corollary 3. Choosing $q_{1}=1$ and $q_{2}=q$ gives nonsymmetrical case as

$$
\begin{aligned}
& {[n]_{1,1}=n,[n]_{1, q}=[n]_{q, 1}=[n]_{q},[n]_{q, q}=n q^{n-1},} \\
& D_{1,1}=\frac{d}{d x}, D_{1, q}=D_{q, 1}=\frac{1-M_{q}}{(1-q)^{X}}, D_{q, q}=M_{q} \frac{d}{d x} .
\end{aligned}
$$

Corollary 4. Taking $q_{1}=q$ and $q_{2}=\frac{1}{q} \quad$ gives symmetrical case as

$$
\begin{aligned}
& {[n]_{q, q}=n q^{n-1},[n]_{1, \frac{1}{q}}=[n]_{\tilde{q}},[n]_{\frac{1}{q}, \frac{1}{q}}=n\left(\frac{1}{q}\right)^{n-1},} \\
& D_{q, q}=M_{q} \frac{d}{d x}, D_{\frac{1}{q}, q}=D_{q, \frac{1}{q}}=\frac{M_{\frac{1}{q}}-M_{q}}{\left(\frac{1}{q}-q\right)^{x}},
\end{aligned}
$$

$D_{\frac{1}{q}, \frac{1}{q}}=M_{\frac{1}{q}} \frac{d}{d x}$. 
The multiple $q$-analogue of $(x-a)^{n}$ is the polynomial

$$
(x+a)_{q_{i}, q_{j}}^{n}:=\left\{\begin{array}{cc}
{\left[\left(x+q_{i}^{n-1} a\right)\left(x+q_{i}^{n-2} q_{j} a\right) \ldots\right.} \\
\left(x+q_{i} q_{j}^{n-2} a\right)\left(x+q_{j}^{n-2} a\right)
\end{array}\right], \text { if } n \geq 1
$$

or equivalently

$$
(x+a)_{q_{i}, q_{j}}^{n}=\sum_{k=0}^{n}\left[\begin{array}{l}
n \\
k
\end{array}\right]_{q_{i}, q_{j}}\left(q_{i} q_{j}\right)^{\frac{k(k-1)}{2}} x^{n-k} a^{k}
$$

where $x$ and $\mathrm{a}$ is commutative, $x a=a x$. $q$-multiple Binomial coefficients and multiple $q$-factorial are defined by

$$
\begin{aligned}
& {[n]_{q_{i}, q_{j}} !} \\
& =\left\{\begin{array}{ll}
{[n]_{q_{i}, q_{j}}[n-1]_{q_{i}, q_{j}} \ldots[2]_{q_{i}, q_{j}}[1]_{q_{i}, q_{j}}} & \text { if } n \geq 1 \\
1 & \text { if } n \geq 1
\end{array}(n \in \mathbb{N}) .\right.
\end{aligned}
$$

Two types of multiple $q$-exponential functions are de.ned by

$$
\begin{aligned}
& e_{q_{i}, q_{j}}(x)=\sum_{n=0}^{\infty} \frac{x^{n}}{[n]_{q_{i}, q_{j}} !} \\
& E_{q_{i}, q_{j}}(x)=\sum_{n=0}^{\infty}\left(q_{i} q_{j}\right)^{\frac{n(n-1)}{2}} \frac{x^{n}}{[n]_{q_{i}, q_{j}} !}
\end{aligned}
$$

which satisfy the following condition for commutative $x$ and $y, x y=y x$

$$
e_{q_{i}, q_{j}}(x+y)_{q_{i} q_{j}}=e_{q_{i}, q_{j}}(x) E_{q_{i}, q_{j}}(y) .
$$

The generalization of Jackson's integral (called multiple $q$-integral) is given by

$$
\int f\left(\frac{x}{q_{i}}\right) d_{\frac{q_{j}}{q_{i}}} x=\left(q_{i}-q_{j}\right) \sum_{k=0}^{\infty} \frac{q_{j}^{k} x}{q_{i}^{k+1}} f\left(\frac{q_{j}^{k}}{q_{i}^{k+1}} x\right) .
$$

Let $f(x)=\sum_{k=0}^{\infty} a_{k} x^{k}$ be formal power series. Applying multiple $q$-integral to the both sides of $f(x)$ gives

$$
\int f\left(\frac{x}{q_{i}}\right) d_{\frac{q_{j}}{q_{i}}} x=\sum_{k=0}^{\infty} q_{i}^{k+1} a_{k} \frac{x^{k+1}}{[k+1]_{q_{i}, q_{j}}}+C
$$

where $C$ is constant.

In the next section, we will use Nalci and Pashaev's method in order to find a systematic study of new types of the Bernoulli polynomials, Euler polynomials and Genocchi polynomials. Also we will obtain recursive formulas for these polynomials.

\section{Main Results}

Recently, analogues of Bernoulli, Euler and Genocchi polynomials were studied by many mathematicians
$[1,2,5,6,11,12,13,17,18,19,20,21]$. We are now ready to give the definition of generating functions, corresponding to multiple $q$-calculus, of Bernoulli type, Euler type and Genocchi type polynomials.

Definition 1. Let $n$ be positive integer, we define

$$
\begin{aligned}
& \mathcal{S}\left(x, z: q_{i}, q_{j}\right)=\sum_{n=0}^{\infty} \mathcal{B}_{n}\left(x: q_{i}, q_{j}\right) \frac{z^{n}}{[n]_{q_{i}, q_{j}} !} \\
& =\frac{z}{e_{q_{i}, q_{j}}(z)-1} e_{q_{i}, q_{j}}(x z) \quad(|z|<2 \pi) \\
& \mathcal{U}\left(x, z: q_{i}, q_{j}\right)=\sum_{n=0}^{\infty} \mathcal{E}_{n}\left(x: q_{i}, q_{j}\right) \frac{z^{n}}{[n]_{q_{i}, q_{j}} !} \\
& =\frac{[2]_{q_{i}, q_{j}}}{e_{q_{i}, q_{j}}(z)+1} e_{q_{i}, q_{j}}(x z) \quad(|z|<\pi) \\
& \mathcal{M}\left(x, z: q_{i}, q_{j}\right)=\sum_{n=0}^{\infty} \mathcal{G}_{n}\left(x: q_{i}, q_{j}\right) \frac{z^{n}}{[n]_{q_{i}, q_{j}}} ! \\
& =\frac{[2]_{q_{i}, q_{j}} z}{e_{q_{i}, q_{j}}(z)+1} e_{q_{i}, q_{j}}(x z) \quad(|z|<\pi)
\end{aligned}
$$

where $\mathcal{B}_{n}\left(x: q_{i}, q_{j}\right), \mathcal{E}_{n}\left(x: q_{i}, q_{j}\right)$ and $\mathcal{G}_{n}\left(x: q_{i}, q_{j}\right)$ are called, respectively, Bernoulli-type, Euler-type and Genocchi-type polynomials.

Corollary 5. Taking $q_{i}=q_{j}=1$ for indexes $i$ and $j$ in the case $N=1$ in Definition 1, we have

$$
\begin{aligned}
& \sum_{n=0}^{\infty} B_{n}(x) \frac{z^{n}}{n !}=\frac{z}{e^{z}-1} e^{x z} \quad(|z|<2 \pi) \\
& \sum_{n=0}^{\infty} E_{n}(x) \frac{z^{n}}{n !}=\frac{2}{e^{z}+1} e^{x z} \quad(|z|<\pi) \\
& \sum_{n=0}^{\infty} G_{n}(x) \frac{z^{n}}{n !}=\frac{2 z}{e^{z}+1} e^{x z} \quad(|z|<\pi)
\end{aligned}
$$

where $B_{n}(x), E_{n}(x)$ and $G_{n}(x)$ are called Bernoulli polynomials, Euler polynomials and Genocchi polynomials, respectively (see $[4,7,14,15,19])$.

Corollary 6. As a special case of Definition 1, we have

$$
\begin{aligned}
& \sum_{n=0}^{\infty} B_{n}(x \mid q) \frac{z^{n}}{[n]_{q} !}=\frac{z}{e_{q}(z)-1} e_{q}(x z)(|z|<2 \pi) \\
& \sum_{n=0}^{\infty} E_{n}(x \mid q) \frac{z^{n}}{[n]_{q} !}=\frac{[2]_{q}}{e_{q}(z)+1} e_{q}(x z)(|z|<\pi) \\
& \sum_{n=0}^{\infty} G_{n}(x \mid q) \frac{z^{n}}{[n]_{q} !}=\frac{[2]_{q} z}{e_{q}(z)+1} e_{q}(x z)(|z|<\pi)
\end{aligned}
$$

where $B_{n}(x \mid q), E_{n}(x \mid q)$ and $G_{n}(x \mid q)$ are called $q$-Bernoulli polynomials, q-Euler polynomials and q-Genocchi polynomials, respectively (see [18,20,21]).

Taking $x=0$ in the above definition, we have

$\mathcal{B}_{n}\left(0: q_{i}, q_{j}\right):=\mathcal{B}_{n}\left(q_{i}, q_{j}\right) \quad$ (Bernoulli-type number) 


$$
\begin{array}{ll}
\mathcal{E}_{n}\left(0: q_{i}, q_{j}\right):=\mathcal{E}_{n}\left(q_{i}, q_{j}\right) & \text { (Euler-type number) } \\
\mathcal{G}_{n}\left(0: q_{i}, q_{j}\right):=\mathcal{G}_{n}\left(q_{i}, q_{j}\right) & \text { (Genocchi-type number) }
\end{array}
$$

and from the above, we write

$$
\begin{aligned}
& \mathcal{S}\left(0, z: q_{i}, q_{j}\right):=\mathcal{S}\left(z: q_{i}, q_{j}\right), \\
& \mathcal{U}\left(0, z: q_{i}, q_{j}\right):=\mathcal{U}\left(z: q_{i}, q_{j}\right), \\
& \mathcal{M}\left(0, z: q_{i}, q_{j}\right):=\mathcal{M}\left(z: q_{i}, q_{j}\right) .
\end{aligned}
$$

From Definition 1 and (2.1), we get the following corollary. Corollary 7. The following functional equations hold true:

$$
\begin{aligned}
& \mathcal{S}\left(x, z: q_{i}, q_{j}\right):=\mathcal{S}\left(z: q_{i}, q_{j}\right) e_{q_{i}, q_{j}}(x z), \\
& \mathcal{U}\left(x, z: q_{i}, q_{j}\right):=\mathcal{U}\left(z: q_{i}, q_{j}\right) e_{q_{i}, q_{j}}(x z), \\
& \mathcal{M}\left(x, z: q_{i}, q_{j}\right):=\mathcal{M}\left(z: q_{i}, q_{j}\right) e_{q_{i}, q_{j}}(x z) .
\end{aligned}
$$

By using Definition 1 and Corollary 7, it becomes

$$
\begin{aligned}
& \sum_{n=0}^{\infty} \mathcal{B}_{n}\left(x: q_{i}, q_{j}\right) \frac{z^{n}}{[n]_{q_{i}, q_{j}} !} \\
& =\left(\sum_{n=0}^{\infty} \mathcal{B}_{n}\left(q_{i}, q_{j}\right) \frac{z^{n}}{[n]_{q_{i}, q_{j}} !}\right)\left(\sum_{n=0}^{\infty} x^{n} \frac{z^{n}}{[n]_{q_{i}, q_{j}} !}\right) .
\end{aligned}
$$

From the rule of Cauchy product, we get

$$
\begin{aligned}
& \sum_{n=0}^{\infty} \mathcal{B}_{n}\left(x: q_{i}, q_{j}\right) \frac{z^{n}}{[n]_{q_{i}, q_{j}} !} \\
& =\sum_{n=0}^{\infty}\left(\sum_{k=0}^{\infty}\left[\begin{array}{l}
n \\
k
\end{array}\right]_{q_{i}, q_{j}} \mathcal{B}_{k}\left(q_{i}, q_{j}\right) x^{n-k}\right) \frac{z^{n}}{[n]_{q_{i}, q_{j}} !} .
\end{aligned}
$$

Comparing the coefficients of $\frac{z^{n}}{[n]_{q_{i}, q_{j}} !}$ in (2.2), we have

$$
\mathcal{B}_{n}\left(x: q_{i}, q_{j}\right)=\sum_{k=0}^{\infty}\left[\begin{array}{l}
n \\
k
\end{array}\right]_{q_{i}, q_{j}} \mathcal{B}_{k}\left(q_{i}, q_{j}\right) x^{n-k}
$$

From this, we can get similar identities for Euler-type and Genocchi-type polynomials. Therefore, we state the following theorem.

Theorem 1. The following identities hold true:

$$
\begin{aligned}
& \mathcal{B}_{n}\left(x: q_{i}, q_{j}\right)=\sum_{k=0}^{\infty}\left[\begin{array}{l}
n \\
k
\end{array}\right]_{q_{i}, q_{j}} \mathcal{B}_{k}\left(q_{i}, q_{j}\right) x^{n-k}, \\
& \mathcal{E}_{n}\left(x: q_{i}, q_{j}\right)=\sum_{k=0}^{\infty}\left[\begin{array}{l}
n \\
k
\end{array}\right]_{q_{i}, q_{j}} \mathcal{E}_{k}\left(q_{i}, q_{j}\right) x^{n-k}, \\
& \mathcal{G}_{n}\left(x: q_{i}, q_{j}\right)=\sum_{k=0}^{\infty}\left[\begin{array}{l}
n \\
k
\end{array}\right]_{q_{i}, q_{j}} \mathcal{G}_{k}\left(q_{i}, q_{j}\right) x^{n-k} .
\end{aligned}
$$

Now we are in a position to investigate some properties of Bernoulli-type numbers and polynomials, Euler-type numbers and polynomials and Genocchi-type numbers and polynomials as follows.
From Definition 1 and by using Cauchy product, we get

$$
\begin{aligned}
& \frac{z}{e_{q_{i}, q_{j}}(z)-1} e_{q_{i}, q_{j}}(z)=z+\frac{z}{e_{q_{i}, q_{j}}(z)-1} \\
& \left(\sum_{n=0}^{\infty} \mathcal{B}_{n}\left(q_{i}, q_{j}\right) \frac{z^{n}}{[n]_{q_{i}, q_{j}} !}\right)\left(\sum_{n=0}^{\infty} \frac{z^{n}}{[n]_{q_{i}, q_{j}} !}\right) \\
& =z+\sum_{n=0}^{\infty} \mathcal{B}_{n}\left(q_{i}, q_{j}\right) \frac{z^{n}}{[n]_{q_{i}, q_{j}} !} \\
& \sum_{n=0}^{\infty}\left(\sum_{k=0}^{\infty}\left[\begin{array}{l}
n \\
k
\end{array}\right]_{q_{i}, q_{j}} \mathcal{B}_{n}\left(q_{i}, q_{j}\right)\right. \\
& =z+\sum_{n=0}^{\infty} \mathcal{B}_{n}\left(q_{i}, q_{j}\right) \frac{z^{n}}{[n]_{q_{i}, q_{j}} !}
\end{aligned}
$$

If we compute both of side and then compare coefficent of $\frac{z^{n}}{[n]_{q_{i}, q_{j}} \text { ! }}$, then for $n>1$, we acquire

$$
\begin{gathered}
\sum_{k=0}^{\infty} \frac{\mathcal{B}_{k}\left(q_{i}, q_{j}\right)}{[k]_{q_{i}, q_{j}} ![n-k]_{q_{i}, q_{j}} !}-\frac{\mathcal{B}_{n}\left(q_{i}, q_{j}\right)}{[n]_{q_{i}, q_{j}} !}=0 \\
\sum_{k=0}^{\infty}\left[\begin{array}{l}
n \\
k
\end{array}\right]_{q_{i}, q_{j}} \mathcal{B}_{k}\left(q_{i}, q_{j}\right)=\mathcal{B}_{n}\left(q_{i}, q_{j}\right) .
\end{gathered}
$$

From this, we can get similar identities for Euler-type numbers and Genocchi-type numbers. The following theorem is an immediate consequence of Eq. (2.4).

Theorem 2. (Recurrence Formula) For $n>1$, we have

$\sum_{k=0}^{n-1}\left[\begin{array}{l}n \\ k\end{array}\right]_{q_{i}, q_{j}} \mathcal{B}_{k}\left(q_{i}, q_{j}\right)= \begin{cases}1, & n=1 \\ 0, & n>1\end{cases}$

$\sum_{k=0}^{\infty}\left[\begin{array}{l}n \\ k\end{array}\right]_{q_{i}, q_{j}} \mathcal{E}_{k}\left(q_{i}, q_{j}\right)+\mathcal{E}_{n}\left(q_{i}, q_{j}\right)= \begin{cases}{[2]_{q_{i}, q_{j}},} & n=0 \\ 0, & n \geq 1\end{cases}$

$\sum_{k=0}^{\infty}\left[\begin{array}{l}n \\ k\end{array}\right]_{q_{i}, q_{j}} \mathcal{G}_{k}\left(q_{i}, q_{j}\right)+\mathcal{G}_{n}\left(q_{i}, q_{j}\right)=\left\{\begin{array}{ll}{[2]_{q_{i}, q_{j}},} & n=1 \\ 0, & n>1\end{array}\right.$.

It is not diffucult to show the following equality:

$$
\left[\begin{array}{l}
n \\
k
\end{array}\right]_{q_{i}, q_{j}}\left[\begin{array}{c}
n-k \\
m
\end{array}\right]_{q_{i}, q_{j}}=\left[\begin{array}{c}
n \\
m+k
\end{array}\right]_{q_{i}, q_{j}}\left[\begin{array}{c}
m+k \\
m
\end{array}\right]_{q_{i}, q_{j}}
$$

By (2.5), we get readily the following theorem.

Theorem 3. For $n \in \mathbb{N}$, the followings hold true

$$
\begin{aligned}
& \mathcal{B}_{n}\left(x+y: q_{i}, q_{j}\right)=\sum_{k=0}^{n}\left[\begin{array}{l}
n \\
k
\end{array}\right]_{q_{i}, q_{j}} \mathcal{B}_{k}\left(x: q_{i}, q_{j}\right) y^{n-k}, \\
& \mathcal{E}_{n}\left(x+y: q_{i}, q_{j}\right)=\sum_{k=0}^{n}\left[\begin{array}{l}
n \\
k
\end{array}\right]_{q_{i}, q_{j}} \mathcal{E}_{k}\left(x: q_{i}, q_{j}\right) y^{n-k}, \\
& \mathcal{G}_{n}\left(x+y: q_{i}, q_{j}\right)=\sum_{k=0}^{n}\left[\begin{array}{l}
n \\
k
\end{array}\right]_{q_{i}, q_{j}} \mathcal{G}_{k}\left(x: q_{i}, q_{j}\right) y^{n-k} .
\end{aligned}
$$


Proof. If we change $x$ by $x+y$ in $\mathcal{S}_{n}\left(x, z: q_{i}, q_{j}\right)$, then we acquire

$$
\begin{aligned}
\sum_{n=0}^{\infty} \mathcal{B}_{n}\left(x+y: q_{i}, q_{j}\right) \frac{z^{n}}{[n]_{q_{i}, q_{j}} !} \\
=\frac{z}{e_{q_{i}, q_{j}}(z)-1} e_{q_{i}, q_{j}}((x+y) z) \\
=\sum_{n=0}^{\infty} \mathcal{B}_{n}\left(q_{i}, q_{j}\right) \frac{z^{n}}{[n]_{q_{i}, q_{j}} !} \sum_{n=0}^{\infty} \frac{z^{n}}{[n]_{q_{i}, q_{j}} !} \\
=\sum_{n=0}^{\infty}\left(\sum _ { k = 0 } ^ { \infty } \left[\begin{array}{l}
n \\
\left.k]_{q_{i}, q_{j}} \mathcal{B}_{n}\left(x: q_{i}, q_{j}\right) y^{n-k}\right) \frac{z^{n}}{[n]_{q_{i}, q_{j}} !}
\end{array}\right.\right.
\end{aligned}
$$

By computing the coefficient $\frac{z^{n}}{[n]_{q_{i}, q_{j}} \text { ! }}$ of both of side, then we have

$$
\mathcal{B}_{n}\left(x+y: q_{i}, q_{j}\right)=\sum_{k=0}^{\infty}\left[\begin{array}{l}
n \\
k
\end{array}\right]_{q_{i}, q_{j}} \mathcal{B}_{n}\left(x: q_{i}, q_{j}\right) y^{n-k} .
$$

The others can be proved in a like manner.

Now we consider the special cases of Theorem 3 as Corollary 8 and Corollary 9.

Corollary 8. Letting $y=1$ in the Theorem 3 , we then get

$$
\begin{aligned}
& \mathcal{B}_{n}\left(x+1: q_{i}, q_{j}\right)=\sum_{k=0}^{\infty}\left[\begin{array}{l}
n \\
k
\end{array}\right]_{q_{i}, q_{j}} \mathcal{B}_{k}\left(x: q_{i}, q_{j}\right), \\
& \mathcal{E}_{n}\left(x+1: q_{i}, q_{j}\right)=\sum_{k=0}^{\infty}\left[\begin{array}{l}
n \\
k
\end{array}\right]_{q_{i}, q_{j}} \mathcal{E}_{k}\left(x: q_{i}, q_{j}\right), \\
& \mathcal{G}_{n}\left(x+1: q_{i}, q_{j}\right)=\sum_{k=0}^{\infty}\left[\begin{array}{l}
n \\
k
\end{array}\right]_{q_{i}, q_{j}} \mathcal{G}_{k}\left(x: q_{i}, q_{j}\right) .
\end{aligned}
$$

Corollary 9. Letting $x=0$ in the Theorem 3, we then get

$$
\begin{aligned}
& \mathcal{B}_{n}\left(x: q_{i}, q_{j}\right)=\sum_{k=0}^{\infty}\left[\begin{array}{l}
n \\
k
\end{array}\right]_{q_{i}, q_{j}} \mathcal{B}_{k}\left(q_{i}, q_{j}\right) x^{n-k}, \\
& \mathcal{E}_{n}\left(x: q_{i}, q_{j}\right)=\sum_{k=0}^{\infty}\left[\begin{array}{l}
n \\
k
\end{array}\right]_{q_{i}, q_{j}} \mathcal{E}_{k}\left(q_{i}, q_{j}\right) x^{n-k}, \\
& \mathcal{G}_{n}\left(x: q_{i}, q_{j}\right)=\sum_{k=0}^{\infty}\left[\begin{array}{l}
n \\
k
\end{array}\right]_{q_{i}, q_{j}} \mathcal{G}_{k}\left(q_{i}, q_{j}\right) x^{n-k} .
\end{aligned}
$$

Theorem 4. The following expressions hold true for $n \in \mathbb{N}$

$$
\begin{aligned}
& \mathcal{B}_{n}\left(x+1: q_{i}, q_{j}\right)-\mathcal{B}_{n}\left(x: q_{i}, q_{j}\right)=[n]_{q_{i}, q_{j}} x^{n-1} \\
& \mathcal{E}_{n}\left(x+1: q_{i}, q_{j}\right)+\mathcal{E}_{n}\left(x: q_{i}, q_{j}\right)+=[2]_{q_{i}, q_{j}} x^{n} \\
& \mathcal{G}_{n}\left(x+1: q_{i}, q_{j}\right)+\mathcal{G}_{n}\left(x: q_{i}, q_{j}\right)=[2]_{q_{i}, q_{j}}[n]_{q_{i}, q_{j}} x^{n-1} .
\end{aligned}
$$

Proof. By using definitions of these polynomials and numbers, one can easily obtain these relations.

Theorem 5. (Identity of Symmetry) The followings hold true for $n \in \mathbb{N}$ :

$$
\begin{aligned}
& \mathcal{B}_{n}\left(1-x: q_{i}, q_{j}\right)=(-1)^{n} \mathcal{B}_{n}\left(x: q_{i}, q_{j}\right), \\
& \mathcal{E}_{n}\left(1-x: q_{i}, q_{j}\right)=(-1)^{n} \mathcal{E}_{n}\left(x: q_{i}, q_{j}\right), \\
& \mathcal{G}_{n}\left(1-x: q_{i}, q_{j}\right)=(-1)^{n+1} \mathcal{G}_{n}\left(x: q_{i}, q_{j}\right) .
\end{aligned}
$$

Proof. Setting $1-x$ instead of $x$ in $\mathcal{S}_{n}\left(x, z: q_{i}, q_{j}\right)$, we then get

$$
\begin{aligned}
& \frac{z}{e_{q_{i}, q_{j}}(z)-1} e_{q_{i}, q_{j}}((1-x) z) \\
& =\sum_{n=0}^{\infty} \mathcal{B}_{n}\left(1-x: q_{i}, q_{j}\right) \frac{z^{n}}{[n]_{q_{i}, q_{j}} !} \\
& =\frac{(-z)}{e_{q_{i}, q_{j}}(-z)-1} e_{q_{i}, q_{j}}(x(-z)) \\
& =\sum_{n=0}^{\infty}(-1)^{n} \mathcal{B}_{n}\left(x: q_{i}, q_{j}\right) \frac{z^{n}}{[n]_{q_{i}, q_{j}}} !
\end{aligned}
$$

Compairing coefficients $\frac{z^{n}}{[n]_{q_{i}, q_{j}} \text { ! }}$ both of side in above equality, we have desired the result. Similar to that of this proof, it can be proved for Euler-type polynomials and Genocchi-type polynomials. So we completed this proof.

Theorem 6. (Raabe's Formula) For $n \in \mathbb{N}$, the followings hold true

$$
\begin{aligned}
& \mathcal{B}_{n}\left(d x: q_{i}, q_{j}\right)=d^{n-1} \sum_{k=0}^{d-1} \mathcal{B}_{n}\left(x+\frac{k}{d}: q_{i}, q_{j}\right) \quad\left(d \in \mathbb{Z}^{+}\right) \\
& \mathcal{E}_{n}\left(d x: q_{i}, q_{j}\right) \\
& =d^{n} \sum_{k=0}^{d-1}(-1)^{k} \mathcal{E}_{n}\left(x+\frac{k}{d}: q_{i}, q_{j}\right) \quad(\mathrm{d} \equiv 1(\bmod 2)) \\
& \mathcal{G}_{n}\left(d x: q_{i}, q_{j}\right) \\
& =d^{n-1} \sum_{k=0}^{d-1}(-1)^{k} \mathcal{G}_{n}\left(x+\frac{k}{d}: q_{i}, q_{j}\right) \quad(\mathrm{d} \equiv 1(\bmod 2)) .
\end{aligned}
$$

Proof. By using Definition 1, then we have

$$
\begin{aligned}
& \sum_{n=0}^{\infty}\left(d^{n-1} \sum_{k=0}^{d-1} \mathcal{B}_{n}\left(x+\frac{k}{d}: q_{i}, q_{j}\right)\right) \frac{z^{n}}{[n]_{q_{i}, q_{j}} !} \\
= & \frac{1}{d} \sum_{k=0}^{d-1} \sum_{n=0}^{\infty} \mathcal{B}_{n}\left(x+\frac{k}{d}: q_{i}, q_{j}\right) \frac{(d z)^{n}}{[n]_{q_{i}, q_{j}} !} \\
= & \frac{1}{d} \sum_{k=0}^{d-1} \frac{d z}{e_{q_{i}, q_{j}}(d z)-1} e_{q_{i}, q_{j}}\left(\left(x+\frac{k}{d}\right) d z\right) \\
= & \frac{z}{e_{q_{i}, q_{j}}(d z)-1} e_{q_{i}, q_{j}}(x d z) \sum_{k=0}^{d-1} e_{q_{i}, q_{j}}(k z) \\
= & \sum_{n=0}^{\infty} \mathcal{B}_{n}\left(d x: q_{i}, q_{j}\right) \frac{z^{n}}{[n]_{q_{i}, q_{j}} !}
\end{aligned}
$$

Similarly, we can prove this theorem for Euler-type numbers and Genocchi-type polynomials. So we omit them. Hence, we complete the proof of this theorem. 
Theorem 7. The three relations between Euler-type numbers and polynomials and Genocchi-type numbers and polynomials are given by

$$
\begin{aligned}
& \mathcal{E}_{n}\left(q_{i}, q_{j}\right)=\frac{\mathcal{G}_{n+1}\left(q_{i}, q_{j}\right)}{n+1}, \\
& \mathcal{E}_{n}\left(x: q_{i}, q_{j}\right)=\frac{\mathcal{G}_{n+1}\left(x: q_{i}, q_{j}\right)}{n+1}, \\
& \mathcal{E}_{n}\left(x: q_{i}, q_{j}\right)=\sum_{k=0}^{\infty}\left[\begin{array}{l}
n \\
k
\end{array}\right]_{q_{i}, q_{j}} \frac{\mathcal{G}_{k+1}\left(q_{i}, q_{j}\right)}{k+1} x^{n-k} .
\end{aligned}
$$

Proof. By Definition 1, we can easily obtain these relations. So we omit the proof.

Let us now apply the multiple $q$-derivative $D_{q_{i}, q_{j}}$, with respect to $x$, on the both sides of Definition 1 ,

$$
\begin{aligned}
& D_{q_{i}, q_{j}}\left(\sum_{n=0}^{\infty} \mathcal{B}_{n}\left(x: q_{i}, q_{j}\right) \frac{z^{n}}{[n]_{q_{i}, q_{j}} !}\right) \\
& =\sum_{n=0}^{\infty} D_{q_{i}, q_{j}} \mathcal{B}_{n}\left(x: q_{i}, q_{j}\right) \frac{z^{n}}{[n]_{q_{i}, q_{j}} !} \\
& =\frac{z}{e_{q_{i}, q_{j}}(z)-1} D_{q_{i}, q_{j}} e_{q_{i}, q_{j}}(x z) \\
& =\frac{z^{2}}{e_{q_{i}, q_{j}}(z)-1} e_{q_{i}, q_{j}}(x z) \\
& =\sum_{n=0}^{\infty} \mathcal{B}_{n}\left(x: q_{i}, q_{j}\right) \frac{z^{n+1}}{[n]_{q_{i}, q_{j}} !} .
\end{aligned}
$$

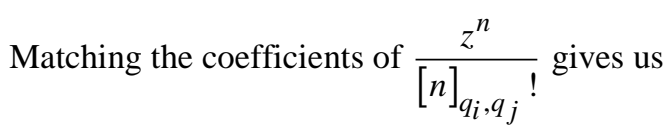

$$
D_{q_{i}, q_{j}} \mathcal{B}_{n}\left(x: q_{i}, q_{j}\right)=[n]_{q_{i}, q_{j}} \mathcal{B}_{n-1}\left(x: q_{i}, q_{j}\right) .
$$

Thus we procure the following theorem.

Theorem 8. The following identities hold true:

$$
\begin{aligned}
& D_{q_{i}, q_{j}} \mathcal{B}_{n}\left(x: q_{i}, q_{j}\right)=[n]_{q_{i}, q_{j}} \mathcal{B}_{n-1}\left(x: q_{i}, q_{j}\right), \\
& D_{q_{i}, q_{j}} \mathcal{E}_{n}\left(x: q_{i}, q_{j}\right)=[n]_{q_{i}, q_{j}} \mathcal{E}_{n-1}\left(x: q_{i}, q_{j}\right)
\end{aligned}
$$

and

$$
D_{q_{i}, q_{j}} \mathcal{G}_{n}\left(x: q_{i}, q_{j}\right)=[n]_{q_{i}, q_{j}} \mathcal{G}_{n-1}\left(x: q_{i}, q_{j}\right) .
$$

Applying $k$-times the operator $D_{q_{i}, q_{j}}$ denoted by $D_{q_{i}, q_{j}}^{k}$ and the limit $t \rightarrow 0$, respectively, to the Definition 1 , we derive that

$$
\mathcal{B}_{n}\left(x: q_{i}, q_{j}\right)=\lim _{t \rightarrow 0} D_{q_{i}, q_{j}}^{k} \frac{z e_{q_{i}, q_{j}}(x z)}{e_{q_{i}, q_{j}}(z)-1} .
$$

So we conclude the following theorem.

Theorem 9. For $k \geq 0$ and $n \geq 0$, we have

$$
\begin{aligned}
& \mathcal{B}_{n}\left(x: q_{i}, q_{j}\right)=\lim _{t \rightarrow 0} D_{q_{i}, q_{j}}^{k} \frac{z e_{q_{i}, q_{j}}(x z)}{e_{q_{i}, q_{j}}(z)-1}, \\
& \mathcal{E}_{n}\left(x: q_{i}, q_{j}\right)=\lim _{t \rightarrow 0} D_{q_{i}, q_{j}}^{k} \frac{[2]_{q_{i}, q_{j}}}{e_{q_{i}, q_{j}}(z)+1} e_{q_{i}, q_{j}}(x z)
\end{aligned}
$$

and

$$
\mathcal{G}_{n}\left(x: q_{i}, q_{j}\right)=\lim _{t \rightarrow 0} D_{q_{i}, q_{j}}^{k} \frac{[2]_{q_{i}, q_{j}}{ }^{z}}{e_{q_{i}, q_{j}}(z)+1} e_{q_{i}, q_{j}}(x z) .
$$

Definition 2. Let $0<a<b$. The definite multiple $q$ integral has the following representation:

$$
\int_{a}^{b} f\left(\frac{x}{q_{i}}\right) d_{\frac{q_{j}}{q_{i}}} x=\left(q_{i}-q_{j}\right) b \sum_{k=0}^{\infty} \frac{q_{j}^{k}}{q_{i}^{k+1}} f\left(\frac{q_{j}^{k}}{q_{i}^{k+1}} b\right)
$$

and

$$
\int_{a}^{b} f\left(\frac{x}{q_{i}}\right) d_{\frac{q_{j}}{q_{i}}} x=\int_{0}^{b} f\left(\frac{x}{q_{i}}\right) d_{\frac{q_{j}}{q_{i}}} x-\int_{0}^{a} f\left(\frac{x}{q_{i}}\right) \frac{d_{q_{j}}}{q_{i}} .
$$

Theorem 10. The following holds true:

$$
\begin{aligned}
& \int_{0}^{b} f\left(\frac{x}{q_{i}}\right) D_{q_{i}, q_{j}} g\left(\frac{x}{q_{i}}\right) d_{\frac{q_{j}}{q_{i}}} x \\
& =\sum_{n=0}^{\infty} f\left(\frac{q_{j}^{k}}{q_{i}^{k+1}} b\right)\left(g\left(\frac{q_{j}^{k} b}{q_{i}^{k}}\right)-g \frac{q_{j}^{k+1} b}{q_{i}^{k+1}}\right) .
\end{aligned}
$$

Proof. From Definition 2, we write that

$$
\begin{aligned}
& \int_{0}^{b} f\left(\frac{x}{q_{i}}\right) D_{q_{i}, q_{j}} g\left(\frac{x}{q_{i}}\right) d_{\frac{q_{j}}{q_{i}}} x \\
& =\left(q_{i}-q_{j}\right) b \sum_{k=0}^{\infty} \frac{q_{j}^{k}}{q_{i}^{k+1}} f\left(\frac{q_{j}^{k}}{q_{i}^{k+1}} b\right) D_{q_{i}, q_{j}} g\left(\frac{q_{j}^{k}}{q_{i}^{k+1}} b\right),
\end{aligned}
$$

where $D_{q_{i}, q_{j}} g\left(\frac{q_{j}^{k}}{q_{i}^{k+1}} b\right)$ equals to

$$
\frac{g\left(\frac{q_{j}^{k}}{q_{i}^{k}} b\right)-g\left(\frac{q_{j}^{k+1}}{q_{i}^{k+1}} b\right)}{\left(q_{i}-q_{j}\right) b} .
$$

Combining the Eq. (2.6) with the Eq. (2.7) gives us the proof of the theorem.

Theorem 11. $\left|\frac{q_{j}}{q_{i}}\right|<1$ and $i, j \in\{1,2, \ldots, N\}$. Then we have

$$
\begin{aligned}
& \int_{0}^{1} \mathcal{B}_{n}\left(\frac{x}{q_{i}}: q_{i}, q_{j}\right) d_{\frac{q_{j}}{q_{i}}} x=\frac{\mathcal{B}_{n+1}\left(1: q_{i}, q_{j}\right)-\mathcal{B}_{n+1}\left(q_{i}, q_{j}\right)}{[n+1]_{q_{i}, q_{j}}} \\
& \int_{0}^{1} \mathcal{E}_{n}\left(\frac{x}{q_{i}}: q_{i}, q_{j}\right) d_{\frac{q_{j}}{q_{i}}} x=\frac{\mathcal{E}_{n+1}\left(1: q_{i}, q_{j}\right)-\mathcal{E}_{n+1}\left(q_{i}, q_{j}\right)}{[n+1]_{q_{i}, q_{j}}}
\end{aligned}
$$


$\int_{0}^{1} \mathcal{G}_{n}\left(\frac{x}{q_{i}}: q_{i}, q_{j}\right) d_{\frac{q_{j}}{q_{i}}} x=\frac{\mathcal{G}_{n+1}\left(1: q_{i}, q_{j}\right)-\mathcal{G}_{n+1}\left(q_{i}, q_{j}\right)}{[n+1]_{q_{i}, q_{j}}}$.

Proof. By using Theorem 1, Definition 2 and for $\left|\frac{q_{j}}{q_{i}}\right|<1$, we have

$$
\begin{aligned}
& \int_{0}^{1} \mathcal{B}_{n}\left(\frac{x}{q_{i}}: q_{i}, q_{j}\right) d_{\frac{q_{j}}{q_{i}}} x \\
& =\sum_{l=0}^{n}\left[\begin{array}{l}
n \\
l
\end{array}\right]_{q_{i}, q_{j}} \mathcal{B}_{n-l}\left(q_{i}, q_{j}\right)\left(\left(q_{i}-q_{j}\right) \sum_{k=0}^{\infty} \frac{q_{j}^{k}}{\left.q_{i}^{k+1}\left(\frac{q_{j}^{k}}{q_{i}^{k+1}}\right)^{l}\right)}\right. \\
& =\sum_{l=0}^{n}\left[\begin{array}{l}
n \\
l
\end{array}\right]_{q_{i}, q_{j}} \mathcal{B}_{n-l}\left(q_{i}, q_{j}\right) \frac{1}{[l+1]_{q_{i}, q_{j}}} \\
& =\frac{\mathcal{B}_{n+1}\left(1: q_{i}, q_{j}\right)-\mathcal{B}_{n+1}\left(q_{i}, q_{j}\right)}{[n+1]_{q_{i}, q_{j}}} .
\end{aligned}
$$

Similarly, the identities of Euler-type polynomials and Genocchi-type polynomials can be shown. Therefore, we complete the proof of theorem.

\section{Further Remarks}

Here we list a few values of Bernoulli-type, Euler-type and Genocchi-type numbers as follows:

\section{Bernoulli-type number:}

Table 1.

\begin{tabular}{|l|}
\hline $\mathcal{B}_{0}\left(q_{i}, q_{j}\right)=1$ \\
\hline $\mathcal{B}_{1}\left(q_{i}, q_{j}\right)=-\frac{1}{[2]_{q_{i}, q_{j}}}$ \\
\hline $\mathcal{B}_{2}\left(q_{i}, q_{j}\right)=-\frac{1}{[3]_{q_{i}, q_{j}}}+\frac{1}{[2]_{q_{i}, q_{j}}}$ \\
$\mathcal{B}_{3}\left(q_{i}, q_{j}\right)=-\frac{1}{[4]_{q_{i}, q_{j}}}+\frac{2}{[2]_{q_{i}, q_{j}}}-\frac{[3]_{q_{i}, q_{j}}}{[2]_{q_{i}, q_{j}}^{2}}$ \\
$\mathcal{B}_{4}\left(q_{i}, q_{j}\right)=-\frac{1}{[5]_{q_{i}, q_{j}}}+\frac{1}{[2]_{q_{i}, q_{j}}}+\frac{[4]_{q_{i}, q_{j}}}{[2]_{q_{i}, q_{j}}}\left(\frac{1}{[3]_{q_{i}, q_{j}}}\right.$ \\
\hline $\left.\left.\begin{array}{c}{[4]_{q_{i}, q_{j}}} \\
{[2]_{q_{i}, q_{j}}}\end{array}\right) \frac{1}{[2]_{q_{i}, q_{j}}}\right)$ \\
\hline A $\left.-\frac{2}{[2]_{q_{i}, q_{j}}}+\frac{[3]_{q_{i}, q_{j}}}{[2]_{q_{i}, q_{j}}^{2}}\right)$
\end{tabular}

As a special case of Table 1 , we get

\begin{tabular}{|l|}
\hline $\mathfrak{B}_{0}(q)=1$ \\
\hline $\mathfrak{B}_{1}(q)=-\frac{1}{[2]_{q}}$ \\
\hline
\end{tabular}

\begin{tabular}{|l|}
\hline $\mathfrak{B}_{2}(q)=-\frac{1}{[3]_{q}}+\frac{1}{[2]_{q}}$ \\
\hline $\mathfrak{B}_{3}(q)=-\frac{1}{[4]_{q}}+\frac{2}{[2]_{q}}-\frac{[3]_{q}}{[2]_{q}^{2}}$ \\
$\mathfrak{B}_{4}(q)=-\frac{1}{[5]_{q}}+\frac{1}{[2]_{q}}+\frac{[4]_{q}}{[2]_{q}}\left(\frac{1}{[3]_{q}}-\frac{1}{[2]_{q}}\right)$ \\
\hline$+\frac{[4]_{q}}{[2]_{q}}\left(\frac{1}{[4]_{q}}-\frac{2}{[2]_{q}}+\frac{[3]_{q}}{[2]_{q}^{2}}\right)$ \\
\hline
\end{tabular}

As a special case of Table 1 , we get

\begin{tabular}{|l|}
\hline$B_{0}=1$ \\
\hline$B_{1}=-\frac{1}{2}$ \\
\hline$B_{2}=\frac{1}{6}$ \\
\hline$B_{3}=0$ \\
\hline$B_{4}=-\frac{1}{30}$ \\
\hline
\end{tabular}

Moreover the first few Bernoulli-type numbers can be shown $N \times N$ matrix with multiple $q$-numbers elements in the following form

$$
\left(\mathcal{B}_{0}\left(q_{i}, q_{j}\right)\right)=\left(\begin{array}{cccc}
1 & 1 & \ldots & 1 \\
1 & 1 & \ldots & 1 \\
\ldots & \ldots & \ldots & \ldots \\
1 & 1 & \ldots & 1
\end{array}\right)
$$$$
\left(\mathcal{B}_{1}\left(q_{i}, q_{j}\right)\right)=\left(\begin{array}{cccc}
-\frac{1}{2 q_{1}} & -\frac{1}{[2]_{q_{1}, q_{2}}} & \ldots & -\frac{1}{[2]_{q_{1}, q_{N}}} \\
-\frac{1}{[2]_{q_{2}, q_{1}}} & -\frac{1}{2 q_{2}} & \ldots & -\frac{1}{[2]_{q_{2}, q_{N}}} \\
\ldots & \ldots & \ldots & \ldots \\
-\frac{1}{[2]_{q_{N}, q_{1}}} & -\frac{1}{[2]_{q_{N}, q_{1}}} & \ldots & -\frac{1}{[2] q_{N}}
\end{array}\right)
$$

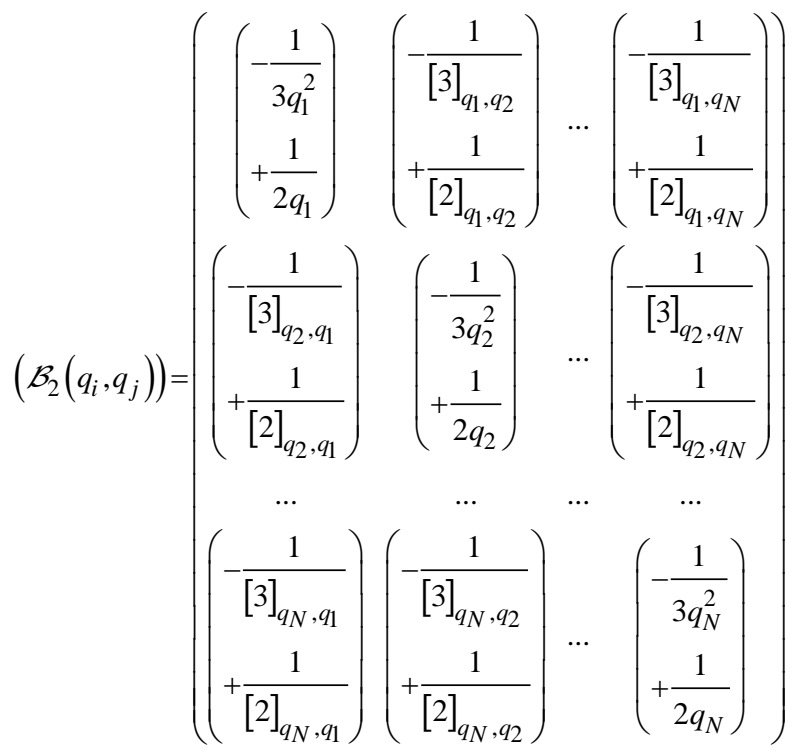


From Definition 1 and the Table 1, we easily acquire the first few Bernoulli-type poly-nomials

Bernoulli-type polynomials

$\mathcal{B}_{0}\left(x: q_{i}, q_{j}\right)=1$

$\mathcal{B}_{1}\left(x: q_{i}, q_{j}\right)=x-\frac{1}{[2]_{q_{i}, q_{j}}}$

$$
\begin{aligned}
& \left(\mathcal{B}_{0}\left(x: q_{i}, q_{j}\right)\right)=\left(\begin{array}{cccc}
1 & 1 & \ldots & 1 \\
1 & 1 & \ldots & 1 \\
\ldots & \ldots & \ldots & \ldots \\
1 & 1 & \ldots & 1
\end{array}\right)=\left(\mathcal{B}_{0}\left(q_{i}, q_{j}\right)\right) \\
& \left(\mathcal{B}_{1}\left(x: q_{i}, q_{j}\right)\right) \\
& =\left(\begin{array}{cccc}
x-\frac{1}{2 q_{1}} & x-\frac{1}{[2]_{q_{1}, q_{2}}} & \ldots & x-\frac{1}{[2]_{q_{1}, q_{N}}} \\
x-\frac{1}{[2]_{q_{2}, q_{1}}} & x-\frac{1}{2 q_{2}} & \ldots & x-\frac{1}{[2]_{q_{2}, q_{N}}} \\
\ldots & \ldots & \ldots & \ldots \\
x-\frac{1}{[2]_{q_{N}, q_{1}}} & x-\frac{1}{[2]_{q_{N}, q_{1}}} & \ldots & x-\frac{1}{[2] q_{N}}
\end{array}\right)
\end{aligned}
$$$$
\mathcal{B}_{2}\left(x: q_{i}, q_{j}\right)=x^{2}-x-\frac{1}{[3]_{q_{i}, q_{j}}}+\frac{1}{[2]_{q_{i}, q_{j}}}
$$

\section{Usual Bernoulli polynomials}

$B_{0}=1$

$B_{1}=x-\frac{1}{2}$

$B_{2}=x^{2}-x-\frac{1}{6}$

Moreover, the first few Bernoulli-type polynomials can be shown $N \times N$ matrix with $q$-numbers elements in the following form

$$
\left(\mathcal{B}_{2}\left(x: q_{i}, q_{j}\right)\right)=\left(\begin{array}{cccc}
x^{2}-x-\frac{1}{3 q_{1}^{2}}+\frac{1}{2 q_{1}} & x^{2}-x-\frac{1}{[3]_{q_{1}, q_{2}}}+\frac{1}{[2]_{q_{1}, q_{2}}} & \ldots & x^{2}-x-\frac{1}{[3]_{q_{1}, q_{N}}}+\frac{1}{[2]_{q_{1}, q_{N}}} \\
x^{2}-x-\frac{1}{[3]_{q_{2}, q_{1}}}+\frac{1}{[2]_{q_{2}, q_{1}}} & x^{2}-x-\frac{1}{3 q_{2}^{2}}+\frac{1}{2 q_{2}} & \ldots & x^{2}-x-\frac{1}{[3]_{q_{2}, q_{N}}}+\frac{1}{[2]_{q_{2}, q_{N}}} \\
\ldots & \ldots & \ldots \\
x^{2}-x-\frac{1}{[3]_{q_{N}, q_{1}}}+\frac{1}{[2]_{q_{N}, q_{1}}} & x^{2}-x-\frac{1}{[3]_{q_{N}, q_{2}}}+\frac{1}{[2]_{q_{N}, q_{2}}} & \ldots & x^{2}-x-\frac{1}{3 q_{N}^{2}}+\frac{1}{2 q_{N}}
\end{array}\right)
$$

\begin{tabular}{|c|c|}
\hline $\mathcal{E}_{0}\left(q_{i}, q_{j}\right)=\frac{[2]_{q_{i}, q_{j}}}{2}$ & \\
\hline $\mathcal{E}_{1}\left(q_{i}, q_{j}\right)=-\frac{[2]_{q_{i}, q_{j}}}{4}$ & \\
\hline $\mathcal{E}_{2}\left(q_{i}, q_{j}\right)=-\frac{[2]_{q_{i}, q_{j}}}{4}+\frac{\left([2]_{q_{i}, q_{j}}\right)^{2}}{8}$ & \\
\hline $\mathcal{E}_{3}\left(q_{i}, q_{j}\right)=-\frac{[2]_{q_{i}, q_{j}}}{4}+\frac{[3]_{q_{i}, q_{j}}[2]_{q_{i}, q_{j}}}{4}\left(1-\frac{[2]_{q_{i}, q_{j}}}{4}\right)$ & \\
\hline $\mathcal{E}_{4}\left(q_{i}, q_{j}\right)=-\frac{[2]_{q_{i}, q_{j}}}{4}+\frac{[4]_{q_{i}, q_{j}}[2]_{q_{i}, q_{j}}}{4}+\frac{[4]_{q_{i}, q_{j}}[3]_{q_{i}, q_{j}}[2]_{q_{i}, q_{j}}}{8}$ & $\frac{1}{[2]_{q_{i}, q_{j}}}-\frac{3}{2}+\frac{[2]_{q_{i}, q_{j}}}{4}$ \\
\hline $\mathcal{E}_{5}\left(q_{i}, q_{j}\right)=-\frac{[2]_{q_{i}, q_{j}}}{4}+\frac{[5]_{q_{i}, q_{j}}[2]_{q_{i}, q_{j}}}{4}+\frac{[5]_{q_{i}, q_{j}}[4]_{q_{i}, q_{j}}[2]_{q_{i}, q_{j}}}{4}$ & $\left.-\frac{3}{4}-\frac{1}{[2]_{q_{i}, q_{j}}}\right)$ \\
\hline$+\frac{[5]_{q_{i}, q_{j}}[4]_{q_{i}, q_{j}}[3]_{q_{i}, q_{j}}[2]_{q_{i}, q_{j}}}{16}\left(\frac{3}{[2]_{q_{i}, q_{j}}}-2+\frac{[2]_{q_{i}, q_{j}}}{4}\right.$ & \\
\hline
\end{tabular}

Euler-type Numbers and Polynomials:

We begin to compute the first few value of $\mathcal{E}_{n}\left(q_{i}, q_{j}\right)$ as follows:

Table 2.

As a special case of Table 2, we have 


\begin{tabular}{|l}
\hline$E_{0}(q)=\frac{[2]_{q}}{2}$ \\
\hline$E_{1}(q)=-\frac{[2]_{q}}{4}$ \\
$E_{2}(q)=-\frac{[2]_{q}}{4}+\frac{\left([2]_{q}\right)^{2}}{8}$ \\
\hline$E_{3}(q)=-\frac{[2]_{q}}{4}+\frac{[3]_{q}[2]_{q}}{4}\left(1-\frac{[2]_{q}}{4}\right)$ \\
\hline$E_{4}(q)=-\frac{[2]_{q}}{4}+\frac{[4]_{q}[2]_{q}}{4}+\frac{[4]_{q}[3]_{q}[2]_{q}}{8}\left(\frac{1}{[2]_{q}}-\frac{3}{2}\right)$ \\
\hline$E_{5}(q)=$ \\
\hline$+\frac{[2]_{q}}{4}+\frac{[5]_{q}[2]_{q}}{4}+\frac{[5]_{q}[4]_{q}[2]_{q}}{4}\left(-\frac{3}{4}-\frac{1}{[2]_{q}}\right)$ \\
$+\frac{[5]_{q}[4]_{q}[3]_{q}[2]_{q}}{16}\left(\frac{3}{[2]_{q}}-2+\frac{[2]_{q}}{4}\right)$
\end{tabular}

As a special case of Table 2, we have

\begin{tabular}{|l|}
\hline$E_{0}(q)=1$ \\
\hline$E_{1}(q)=-\frac{1}{2}$ \\
\hline$E_{2}(q)=0$ \\
\hline$E_{3}(q)=\frac{1}{4}$ \\
\hline$E_{4}(q)=0$ \\
\hline$E_{5}(q)=-\frac{1}{2}$ \\
\hline
\end{tabular}

Moreover the first few Euler-type numbers can be shown $N \times N$ matrix with $q$-numbers elements in the following form

$$
\begin{aligned}
\left(\mathcal{E}_{0}\left(q_{i}, q_{j}\right)\right) & =\left(\begin{array}{cccc}
q_{1} & \frac{[2]_{q_{1}, q_{2}}}{2} & \ldots & \frac{[2]_{q_{1}, q_{N}}}{2} \\
\frac{[2]_{q_{2}, q_{1}}}{2} & q_{2} & \ldots & \frac{[2]_{q_{2}, q_{N}}}{2} \\
\ldots & \ldots & \ldots & \ldots \\
\frac{[2]_{q_{N}, q_{1}}}{2} & \frac{[2]_{q_{N}, q_{2}}}{2} & \ldots & q_{N}
\end{array}\right) \\
\left(\mathcal{E}_{1}\left(q_{i}, q_{j}\right)\right) & =\left(\begin{array}{cccc}
-\frac{q_{1}}{2} & -\frac{[2]_{q_{1}, q_{2}}}{4} & \ldots & -\frac{[2]_{q_{1}, q_{N}}}{4} \\
-\frac{[2]_{q_{2}, q_{1}}}{4} & -\frac{q_{2}}{2} & \ldots & -\frac{[2]_{q_{2}, q_{N}}}{4} \\
\ldots & \ldots & \ldots & \ldots \\
-\frac{[2]_{q_{N}, q_{1}}}{4} & -\frac{[2]_{q_{N}, q_{2}}}{4} & \ldots & -\frac{q_{N}}{2}
\end{array}\right)
\end{aligned}
$$

$$
\left(\mathcal{E}_{2}\left(x: q_{i}, q_{j}\right)\right)
$$

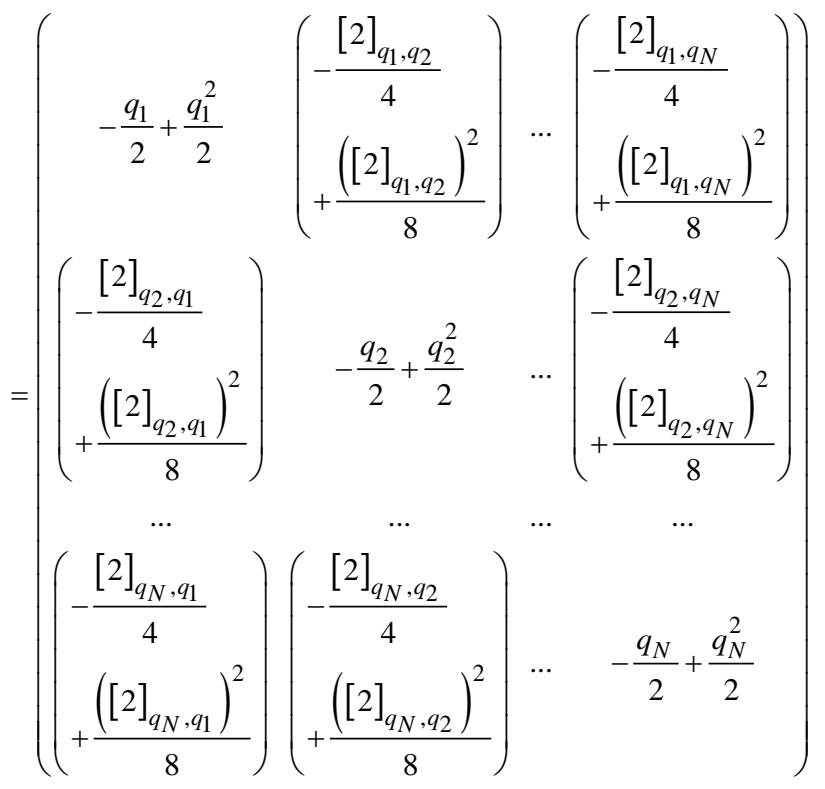

From Definition 1 and the Table 2, we easily acquire the first few Euler-type polynomials

Euler-type polynomials

$$
\begin{aligned}
\mathcal{E}_{0}\left(x: q_{i}, q_{j}\right)= & \frac{[2]_{q_{i}, q_{j}}}{2} \\
\mathcal{E}_{1}\left(x: q_{i}, q_{j}\right)= & \frac{[2]_{q_{i}, q_{j}}}{2} x-\frac{[2]_{q_{i}, q_{j}}}{4} \\
\mathcal{E}_{2}\left(x: q_{i}, q_{j}\right)= & \frac{[2]_{q_{i}, q_{j}}}{2} x^{2}-\frac{\left([2]_{q_{i}, q_{j}}\right)^{2}}{4} x \\
& -\frac{[2]_{q_{i}, q_{j}}}{4}+\frac{\left([2]_{q_{i}, q_{j}}\right)^{2}}{8}
\end{aligned}
$$

\section{Usual Euler polynomials}

$E_{0}=1$

$E_{1}=x-\frac{1}{2}$
$E_{2}=x^{2}-x$

Moreover the first few Euler-type polynomials can be shown $N \times N$ matrix with multiple $q$-numbers elements in the following form

$$
\begin{aligned}
& \left(\mathcal{E}_{0}\left(q_{i}, q_{j}\right)\right) \\
& =\left(\begin{array}{cccc}
q_{1} & \frac{[2]_{q_{1}, q_{2}}}{2} & \ldots & \frac{[2]_{q_{1}, q_{N}}}{2} \\
\frac{[2]_{q_{2}, q_{1}}}{2} & q_{2} & \ldots & \frac{[2]_{q_{2}, q_{N}}}{2} \\
\ldots & \ldots & \ldots & \ldots \\
\frac{[2]_{q_{N}, q_{1}}}{2} & \frac{[2]_{q_{N}, q_{2}}}{2} & \ldots & q_{N}
\end{array}\right)
\end{aligned}
$$


137

Turkish Journal of Analysis and Number Theory

$$
\begin{aligned}
& \left(\mathcal{E}_{1}\left(x: q_{i}, q_{j}\right)\right)=\left(\begin{array}{cccc}
q_{1} x-\frac{q_{1}}{2} & \frac{[2]_{q_{1}, q_{2}}}{2} x-\frac{[2]_{q_{1}, q_{2}}}{4} & \ldots & \frac{[2]_{q_{1}, q_{2}}}{2} x-\frac{[2]_{q_{1}, q_{N}}}{4} \\
\frac{[2]_{q_{1}, q_{2}}}{2} x-\frac{[2]_{q_{2}, q_{1}}}{4} & q_{2} x-\frac{q_{2}}{2} & \ldots & \frac{[2]_{q_{1}, q_{2}}}{2} x-\frac{[2]_{q_{2}, q_{N}}}{4} \\
\ldots & \ldots & \ldots & \ldots \\
\frac{[2]_{q_{1}, q_{2}}}{2} x-\frac{[2]_{q_{N}, q_{1}}}{4} & \frac{[2]_{q_{1}, q_{2}}}{2} x-\frac{[2]_{q_{N}, q_{2}}}{4} & \ldots & q_{N} x-\frac{q_{N}}{2}
\end{array}\right) \\
& \left(\mathcal{E}_{2}\left(x: q_{i}, q_{j}\right)\right)=\left(\begin{array}{ccc}
q_{1} x^{2}-q_{1}^{2} x-\frac{q_{1}}{2}+\frac{q_{1}^{2}}{2} & \ldots & \frac{[2]_{q_{1}, q_{N}}}{2} x^{2}-\frac{\left([2]_{q_{1}, q_{N}}\right)^{2}}{4} x-\frac{[2]_{q_{1}, q_{N}}}{4}+\frac{\left([2]_{q_{1}, q_{N}}\right)^{2}}{8} \\
\frac{[2]_{q_{1}, q_{2}}}{2} x^{2}-\frac{\left([2]_{q_{2}, q_{1}}\right)^{2}}{4} x-\frac{[2]_{q_{1}, q_{2}}}{4}+\frac{\left([2]_{q_{1}, q_{2}}\right)^{2}}{8} & \ldots & \frac{[2]_{q_{2}, q_{N}}}{2} x^{2}-\frac{\left([2]_{q_{2}, q_{N}}\right)^{2}}{4} x-\frac{[2]_{q_{1}, q_{N}}}{4}+\frac{\left([2]_{q_{1}, q_{N}}\right)^{2}}{8} \\
\ldots & \ldots & \ldots \\
\frac{[2]_{q_{N}, q_{1}}}{2} x^{2}-\frac{\left([2]_{q_{N}, q_{1}}\right)^{2}}{4} x-\frac{[2]_{q_{N}, q_{1}}+\frac{\left([2]_{q_{N}, q_{1}}\right)^{2}}{4}}{4} & \ldots & q_{N} x^{2}-q_{N}^{2} x-\frac{q_{N}}{2}+\frac{q_{N}^{2}}{2}
\end{array}\right)
\end{aligned}
$$

Genocchi-type Numbers and Polynomials:

We begin to compute the first few value of $\mathcal{G}_{1}\left(q_{i}, q_{j}\right)$ as follows:

Table 3.

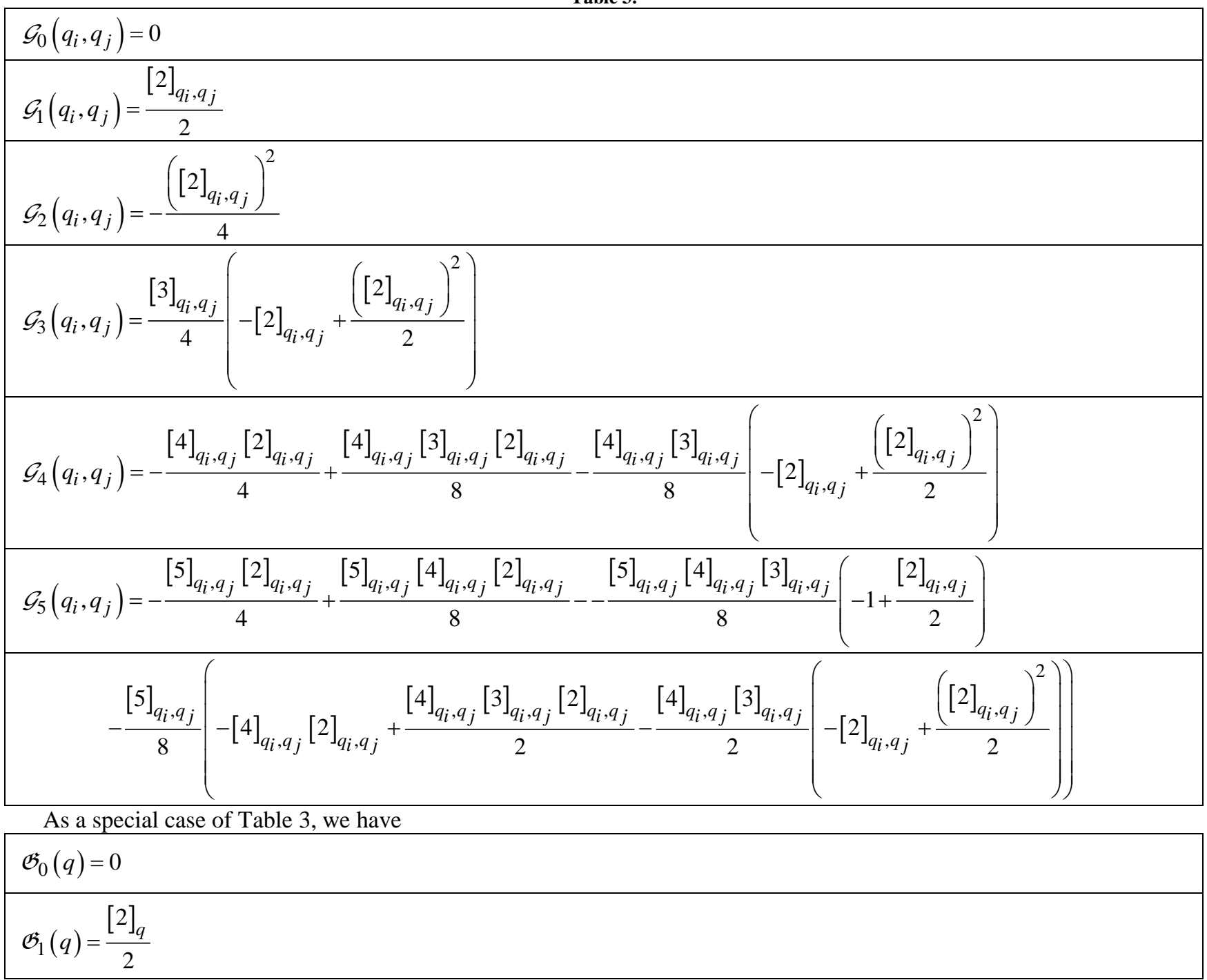




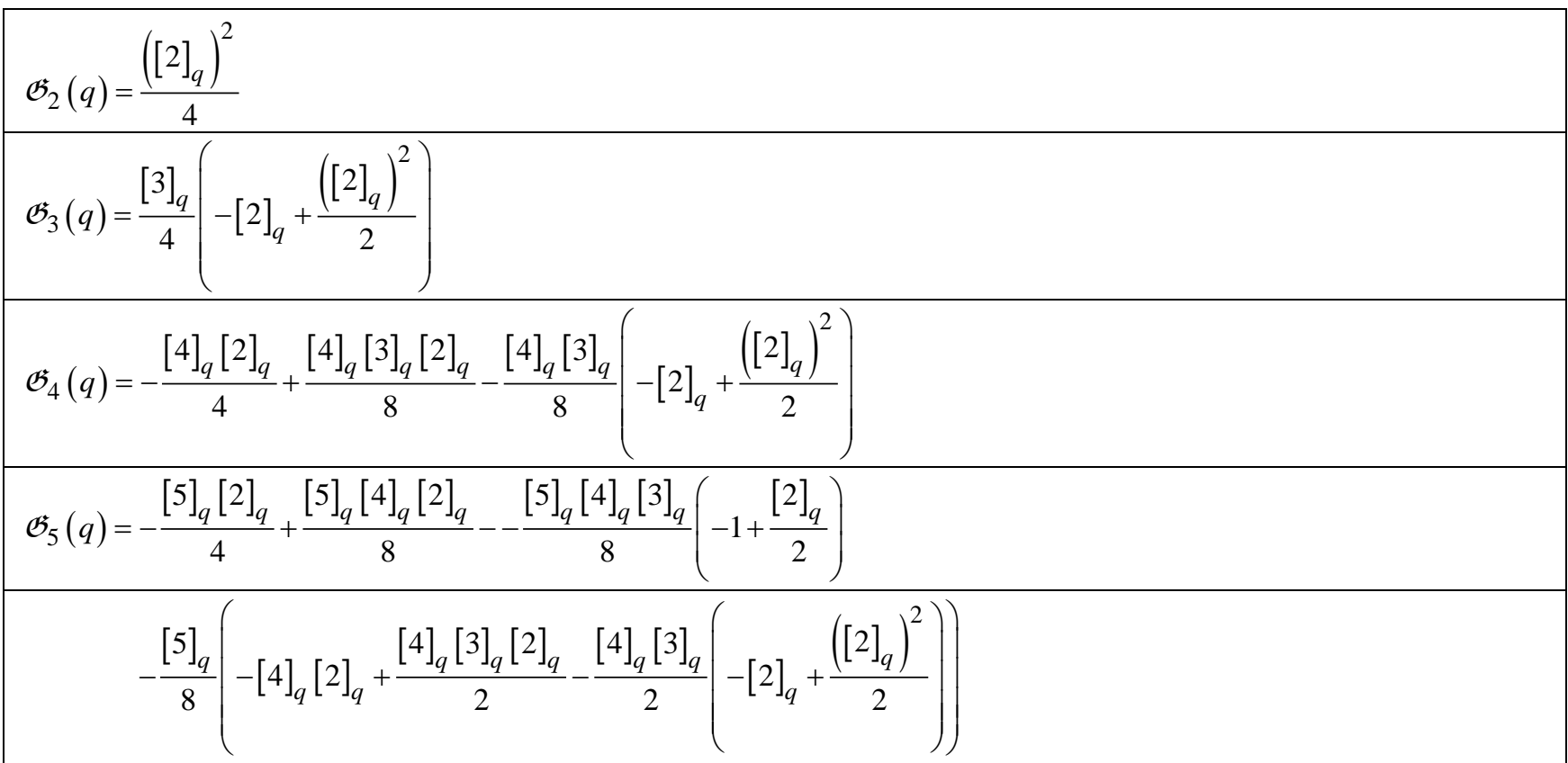

As a special case of Table 3, we have

$$
\begin{array}{|l|}
\hline G_{0}=0 \\
\hline G_{1}=1 \\
\hline G_{2}=-1 \\
\hline G_{3}=0 \\
\hline G_{4}=1 \\
\hline G_{5}=0 \\
\hline
\end{array}
$$

Moreover the first few Genocchi-type numbers can be shown $N \times N$ matrix with multiple $q$-numbers elements in the following form

$$
\begin{aligned}
& \left(\mathcal{G}_{0}\left(q_{i}, q_{j}\right)\right)=\left(\begin{array}{cccc}
0 & 0 & \ldots & 0 \\
0 & 0 & \ldots & 0 \\
\ldots & \ldots & \ldots & \ldots \\
0 & 0 & \ldots & 0
\end{array}\right) \\
& \left(\mathcal{G}_{1}\left(q_{i}, q_{j}\right)\right)=\left(\begin{array}{cccc}
q_{1} & \frac{[2]_{q_{1}, q_{2}}}{2} & \ldots & \frac{[2]_{q_{1}, q_{N}}}{2} \\
\frac{[2]_{q_{2}, q_{1}}}{2} & q_{2} & \ldots & \frac{[2]_{q_{2}, q_{N}}}{2} \\
\ldots & \ldots & \ldots & \ldots \\
\frac{[2]_{q_{N}, q_{1}}}{2} & \frac{[2]_{q_{N}, q_{2}}}{2} & \ldots & q_{N}
\end{array}\right) \\
& \left(\mathcal{G}_{2}\left(q_{i}, q_{j}\right)\right) \\
& =\left(\begin{array}{cccc}
-\frac{q_{1}^{2}}{2} & -\frac{\left([2]_{q_{1}, q_{2}}\right)^{2}}{4} & \ldots & -\frac{\left([2]_{q_{1}, q_{N}}\right)^{2}}{4} \\
-\frac{\left([2]_{q_{2}, q_{1}}\right)^{2}}{4} & -\frac{q_{2}^{2}}{2} & \ldots & -\frac{\left([2]_{q_{2}, q_{N}}\right)^{2}}{4} \\
\ldots & \ldots & \ldots & \ldots \\
-\frac{\left([2]_{q_{N}, q_{1}}\right)^{2}}{4} & -\frac{\left([2]_{q_{N}, q_{2}}\right)^{2}}{4} & \ldots & -\frac{q_{N}^{2}}{2}
\end{array}\right)
\end{aligned}
$$

From Definition 1 and the Table 3, we easily acquire the first few Genocchi-type poly-nomials

\section{Genocchi-type polynomials}

$$
\begin{aligned}
& \mathcal{G}_{0}\left(x: q_{i}, q_{j}\right)=0 \\
& \mathcal{G}_{1}\left(x: q_{i}, q_{j}\right)=\frac{[2]_{q_{i}, q_{j}}}{2} \\
& \mathcal{G}_{2}\left(x: q_{i}, q_{j}\right)=\frac{\left([2]_{q_{i}, q_{j}}\right)^{2}}{2} x-\frac{\left([2]_{q_{i}, q_{j}}\right)^{2}}{4}
\end{aligned}
$$

\section{Usual Genocchi polynomials}

$$
\begin{aligned}
& G_{0}=0 \\
& G_{1}=1 \\
& G_{2}=2 x-1
\end{aligned}
$$

Moreover the first few Genocchi-type polynomials can be shown $N \times N$ matrix with multiple $q$-numbers elements in the following form

$$
\left(\mathcal{G}_{0}\left(q_{i}, q_{j}\right)\right)=\left(\begin{array}{cccc}
0 & 0 & \ldots & 0 \\
0 & 0 & \ldots & 0 \\
\ldots & \ldots & \ldots & \ldots \\
0 & 0 & \ldots & 0
\end{array}\right)
$$

$\left(\mathcal{G}_{1}\left(q_{i}, q_{j}\right)\right)$

$$
=\left(\begin{array}{cccc}
q_{1} & \frac{[2]_{q_{1}, q_{2}}}{2} & \ldots & \frac{[2]_{q_{1}, q_{N}}}{2} \\
\frac{[2]_{q_{2}, q_{1}}}{2} & q_{2} & \ldots & \frac{[2]_{q_{2}, q_{N}}}{2} \\
\ldots & \ldots & \ldots & \ldots \\
\frac{[2]_{q_{N}, q_{1}}}{2} & \frac{[2]_{q_{N}, q_{2}}}{2} & \ldots & q_{N}
\end{array}\right)
$$




$$
\left(\mathcal{G}_{2}\left(x: q_{i}, q_{j}\right)\right)=\left(\begin{array}{ccccc}
2 q_{1}^{2} x-\frac{q_{1}^{2}}{2} & \frac{\left([2]_{q_{1}, q_{2}}\right)^{2}}{2}-\frac{\left([2]_{q_{1}, q_{2}}\right)^{2}}{4} & \ldots & \frac{\left([2]_{q_{1}, q_{N}}\right)^{2}}{2}-\frac{\left([2]_{q_{1}, q_{N}}\right)^{2}}{4} \\
\frac{\left([2]_{q_{2}, q_{1}}\right)^{2}}{2}-\frac{\left([2]_{q_{2}, q_{1}}\right)^{2}}{4} & 2 q_{2}^{2} x-\frac{q_{2}^{2}}{2} & \ldots & \frac{\left([2]_{q_{2}, q_{N}}\right)^{2}}{2}-\frac{\left([2]_{q_{2}, q_{N}}\right)^{2}}{4} \\
\ldots & \ldots & \ldots & \ldots \\
\frac{\left([2]_{q_{N}, q_{1}}\right)^{2}}{2}-\frac{\left([2]_{q_{N}, q_{1}}\right)^{2}}{4} & \frac{\left([2]_{q_{N}, q_{2}}\right)^{2}}{2}-\frac{\left([2]_{q_{N}, q_{2}}\right)^{2}}{4} & \ldots & 2 q_{N}^{2} x-\frac{q_{N}^{2}}{2}
\end{array}\right)
$$

[11] U. Duran, M. Acikgoz, A. Esi, S. Araci, Some new symmetric identities involving q-Genocchi polynomials under $S_{4}$, Journal of Mathematical Analysis, Vol 6, Issue 4 (2015).

\section{References}

[1] G. M. Philips, Bernstein polynomials based on the q-integers, Annals of Numerical Mathematics, vol. 4,no. 1-4, pp. 511-518, 1997.

[2] C. S. Ryoo, A note on q-Bernoulli numbers and polynomials, Appl. Math. Lett. 20 (2007) 524-531.

[3] V. Kac and P. Cheung, Quantum Calculus, New York: Springer, 2002.

[4] G. S. Cheon, A note on the Bernoulli and Euler polynomials, Appl. Math. Lett. 16 (2003) 365-368.

[5] Q. M. Luo, Some results for the q-Bernoulli and q- Euler polynomials, J. Math. Anal. Appl. 363 (2010) 7-18.

[6] H. M. Srivastava, Some generalizations and basic (or q-) extensions of the Bernoulli, Euler and Genocchi polynomials, Appl. Math. Inform. Sci. 5 (2011), 390-444.

[7] H. M. Srivastava, Some formulas for the Bernoulli and Euler polynomials at rational arguments, Math. Proc. Cambridge Philos. Soc. 129 (2000), 77-84.

[8] S. Nalci and O. K. Pashaev, q-Bernoulli Numbers and Zeros of qSine Function, arXiv: 1202,2265v12012.

[9] S. Nalci and O.K. Pashaev, Exactly Solvable q-extended Nonlinear Classical and Quantum Models, Lambert Academic Publishing, 2014.

[10] M. Acikgoz, S. Araci, U.Duran, Research on Some New Theorems and Some Elementary Functions Under Multiple Quantum Calculus, preprint.
[12] U. Duran, M. Acikgoz, S. Araci, Symmetric identities involving weighted $q$-Genocchi polynomials under $S_{4}$, Proceedings of the Jangjeon Mathematical Society,18 (2015), No. 4, pp 455-465.

[13] S. Araci, E. Ağyüz and M. Acikgoz, On a q-analog of some numbers and polynomials, J. Ineq. Appl. (2015) 2015:19.

[14] S. Araci, M. Acikgoz, A. Bagdasaryan, E. Sen, The Legendre Polynomials Associated with Bernoulli, Euler, Hermite and Bernstein Polynomials, Turkish J. Anal. Number Theory, Vol. 1, No. 1, (2013) 1-3.

[15] S. Araci, M. Acikgoz, E. Sen, Some New Formulae for Genocchi Numbers and Polynomials Involving Bernoulli and Euler Polynomials, Int. J. Math. Math. Sci., vol. 2014(2014), Art. ID: 760613, 7pp.

[16] S. Araci, U. Duran, M. Acikgoz, Symmetric Identities Involving qFrobenius-Euler Polynomials under Sym (5), Turkish J. Anal. Number Theory, Vol. 3, No. 3, (2015) pp 90-93.

[17] T. Kim, q-Volkenborn integration, Russ. J. Math. Phys. Vol. 9, no.3, pp. 288-299, 2002.

[18] T. Kim, q-Generalized Euler numbers and polynomials, Russ. J. Math. Phys., Vol. 13, No. 3 (2006), pp. 293.298.

[19] T. Kim, Symmetry p-adic invariant integral on $\mathbb{Z}_{p}$ for Bernoulli and Euler polynomials, J. Differ. Equ. Appl. 14 (2008), 1267-1277.

[20] B. A. Kupershmidt, Reflection Symmetries of q-Bernoulli Polynomials, J. Nonlinear Math. Phys. 12 (Suppl. 1), 412-422 (2005).

[21] N. I. Mahmudov, On a class of q-Bernoulli and Euler polynomials, Advances in Difference Equations 2013, 2013:108. 